\title{
PHOTOMETRIC PROPERTIES OF CP STARS IN THE INFRARED
}

\author{
FRANCESCO A. CATALANO \\ Istituto di Astronomia, Città Universitaria, I-95125 Catania Italy
}

FRANCESCO LEONE

Osservatorio Astrofisico di Catania, Città Universitaria, I-95125 Catania Italy

\section{REINHOLD KROLL}

Institut für Astronomie und Astrophysik Am Hubland, D-8700 Würzburg Germany

\begin{abstract}
Eightysix chemically peculiar stars have been observed in the near infrared with the aim to extend the comprehension of the differences between normal and peculiar stars. These stars have been checked for variability. Most of the stars are previously known light variables, although their periods were not accurate enough to phase together different kinds of observations carried out several years apart. For about 20 stars the period has been refined by combining our own uvby observations with the ones available in the literature.
\end{abstract}

\section{INTRODUCTION}

In recent years there has been a growing interest in the infrared properties of chemically peculiar stars of the upper main sequence (CP stars, according to Preston's (1974) nomenclature). Such an interest derives from the belief that CP stars and their complex atmospheres look much simpler in the infrared. It is in fact generally accepted that at the longer wavelengths the radiation field is normal, due to the absence of any significant ultraviolet flux redistribution and line blocking.

To verify such a hypothesis, we have carried out photometric infrared observations of CP stars.

The observations were carried out in several runs since 1986 in the near IR bands $\mathrm{J}, \mathrm{H}$, and $\mathrm{K}$, mainly at the $1 \mathrm{~m}$ photometric telescope at ESO, La Silla, Chile, using an InSb detector cooled with liquid nitrogen. A detailed description of the ESO infrared photometers can be found in Bouchet (1989). The integration times were selected to give a precision of 0.001 mag. in the measurements. ESO standard software was used for all reduction steps. Magnitudes in the standard IR system were also established by standard stars from the ESO list. To check for variability, closeby comparisons were chosen so as to have as similar colors and brightness as possible. 


\section{OVERALL FLUX DISTRIBUTION}

Indeed it has been shown by Kroll et al. (1987) that for CP stars infrared fluxes and colors in the range 1 to $5 \mu$ are not different from those of normal main sequence stars when compared to a black body. Combining ultraviolet, visible and infrared fluxes, Leone \& Catalano (1991) have shown that CP stars can not be distinguished from normal stars in the plane $V-J$ vs. $V-H$. Effective temperatures, extending Adelman and coworkers (Pyper \& Adelman 1985, and references therein) procedure to the near infrared, are even lower than the ones inferred from the Paschen continuum. Moreover Leone \& Catalano (1991) have shown that the solar composition Kurucz model atmospheres, which are used to fit the CP stars spectra from $\lambda 5500$ to $\lambda 16500 \AA$, give a fair representation of the overall flux distribution, with the exception of the Balmer region, where CP stars appear generally brighter than normal, this excess being just a few percent of the total flux. A straight consequence of a such flux distribution is that the effective temperature deduced by classical method from the visible part of spectrum can be too high and that from the UV and IR regions a value of the effective temperature closer to the real one can be determined.

\section{VARIABILITY}

In spite of this normality of the infrared flux behavior, peculiar abundances and magnetic fields seem to affect the near infrared. Catalano et al. (1991a, hereafter CKL) have shown that, out of the eight CP stars monitored throughout their rotational periods, at least six are certainly variable in the near infrared, although the amplitudes shown are smaller than in the visible. Moreover, CKL have found evidence that within the accuracy of the known ephemeris, the near infrared light curves seem to be phase related to the magnetic field variations in the sense that magnetic field extrema might coincide in time with infrared light extrema, although strong field regions can apparently coincide as well with infrared (primary or secondary) maxima or minima. Up to now 86 stars have been checked for variability in the infrared, but upon looking at the data, we soon discovered that for too many stars of the sample the period is not known within the requested accuracy to allow investigation of the above said phase relation. Since multicolor photometric observations are the most convenient way to determine the period, we have started a research program aimed to accurately determining periods on the basis of new photometric observations.

In Table 1 and 2 we list the stars and the degree of completeness for the observed variations in the infrared using the code:

vv ... well defined light curves;

v: ... definite variations, nearly complete light curves;

v? ... indication of variability: incomplete light curves;

::: ... insufficient data;

?? ... probably constant, although with a large dispersion of the observed points; 
TABLE I Stars observed in the infrared

\begin{tabular}{|c|c|c|c|c|c|}
\hline HD & HR/other & Name & Sp. & $P(d)$ & \\
\hline 36485 & 1851 & $\delta$ Ori C & B2 He & 0.830056 & $\mathbf{v v}$ \\
\hline 37017 & 1890 & V1046 Ori & B2 He & 0.901195 & v? \\
\hline 37479 & 1932 & $\sigma$ Ori $\mathrm{E}$ & B2 He & 1.19081 & $\mathbf{v v}$ \\
\hline 37776 & $B D-1^{\circ} 1005$ & V901 Ori & B3 He & 1.53869 & $\mathrm{v}:$ \\
\hline 58260 & GC 9873 & $\ldots$ & B3 He & 1.657: & $\mathrm{v} ?$ \\
\hline 60344 & $\mathrm{CoD}-23^{\circ} 5673$ & $\ldots$ & B3 He & 1.2: & v? \\
\hline 64740 & 3089 & 216G. Pup & B2 $\mathrm{He}$ & 1.33026 & $\mathrm{v}:$ \\
\hline 184927 & $\mathrm{BD}+30^{\circ} 3645$ & V1671Cyg & B2 He & 9.52973 & $:::$ \\
\hline 5737 & 280 & $\alpha \mathrm{Scl}$ & B6 He wk & 21.647 & $:::$ \\
\hline 21699 & 1063 & V396 Per & B8 $\mathrm{He}$ wk $\mathrm{Si}$ & 2.492 & ::: \\
\hline 28843 & 1441 & DZ Eri & B9 He wk & 1.373813 & $\mathbf{v v}$ \\
\hline 35456 & GC 6661 & $\ldots$ & B7 He wk & 1.8: & $\mathbf{v v}$ \\
\hline 37151 & GC 6961 & V1179 Ori & B8 He wk Si & 0.804404 & v: \\
\hline 49333 & 2509 & HK CMa & B7 $\mathrm{He}$ wk Si & 2.1800 & $\mathbf{v v}$ \\
\hline 74196 & 3448 & 55G. Vel & B7 He wk & $0.3880:$ & $\mathrm{v} ?$ \\
\hline 125823 & 5378 & $a$ Cen & B5 He wk & 8.8171 & $\mathbf{v v}$ \\
\hline 142990 & 5942 & V913 Sco & B6 He wk & 0.4885035 & v: \\
\hline 144334 & 5988 & V929 Sco & B8 He wk & 1.494971 & $\mathbf{v v}$ \\
\hline 175362 & 7129 & V686 Cra & B5 He wk Si & 3.67375 & $\mathbf{v v}$ \\
\hline 12767 & 612 & $\nu$ For & $\mathrm{A} 0 \mathrm{Si}$ & 3.7453 & v: \\
\hline 19832 & 954 & SX Ari & $\mathrm{B} 8 \mathrm{Si}$ & 0.72789761 & v: \\
\hline 22470 & 1100 & EG Eri & B9 Si & 1.92895 & $\mathbf{v v}$ \\
\hline 25267 & 1240 & $\tau^{9}$ Eri & A0 Si & 1.21005 & $\mathbf{v v}$ \\
\hline 29305 & 1465 & $\alpha$ Dor A & A0 Si & 2.943176 & $\mathbf{v v}$ \\
\hline 30466 & GC5857 & V473 Tau & $\mathrm{A} 0 \mathrm{Si}$ & $1.39 ?$ & $:::$ \\
\hline 34452 & 1732 & IQ Aur & B9 Si & 2.466 & $:::$ \\
\hline 40312 & 2095 & $\theta$ Aur & A0 Si & 3.619 & $:::$ \\
\hline 54118 & 2683 & V386 Car & A0 Si & 3.275170 & ?? \\
\hline 56455 & 2761 & PR Pup & $\mathrm{A} 0 \mathrm{Si}$ & 2.06370 & $\mathbf{v v}$ \\
\hline 66255 & 3151 & PY Pup & A0 Si & 6.81780 & $\mathrm{v}$ ? \\
\hline 73340 & 3413 & HV Vel & B9 Si & 2.667588 & $\mathrm{v}$ ? \\
\hline 92664 & 4185 & V364 Car & B9 Si & 1.67309 & $\mathbf{v v}$ \\
\hline 114365 & 4964 & V824 Cen & A0 Si & 1.271925 & $\mathrm{v:}$ \\
\hline 116890 & 5066 & EZ Mus & B9 Si & 4.301176 & $\mathbf{v v}$ \\
\hline 122532 & 5269 & V828 Cen & B9 Si & 3.6807 & $\mathbf{v v}$ \\
\hline 124224 & 5313 & CU Vir & B9 Si & 0.52067688 & $\mathbf{v v}$ \\
\hline 144231 & GC 21650 & LL TrA & B9 Si & 4.39136 & $\mathbf{v}:$ \\
\hline 145102 & GC 21737 & 31G. Sco & B9 Si & 1.417951 & ?? \\
\hline 203585 & 8180 & $\theta^{2} \mathrm{Mic}$ & A0 Si & 0.83: & $\mathrm{v}:$ \\
\hline 215441 & $\mathrm{BD}+54^{\circ} 2846$ & GL Lac & B9 Si & 9.4875 & $:::$ \\
\hline 221006 & 8919 & CG Tuc & A0 Si & 2.31483 & $\mathbf{v v}$ \\
\hline
\end{tabular}


TABLE II Stars observed in the infrared

\begin{tabular}{rcclcc}
\multicolumn{1}{r}{ HD } & HR/other & Name & Sp. & P(d) & \\
& & & & & \\
9996 & 465 & GY And & A0 SiSrEu & 85000 & $:::$ \\
10783 & GC 2141 & UZ Psc & A2 SiCrSr & 4.13281 & v? \\
12447 & 596 & $\alpha^{2}$ Psc & A2 SiSrCr & 1.49070 & v? \\
18296 & 873 & LT Per & A0 SiSr & 2.884 & $::$ \\
25823 & 1268 & GS Tau & B9 SrSi & 7.227 & $:::$ \\
32633 & GC 6222 & HZ Aur & B9 SiCr & 6.430 & $:::$ \\
49606 & 2519 & OV Gem & B8 MnHgSi & $3.35 ?$ & $:::$ \\
71866 & GC 11639 & TZ Lyn & A1 EuSrSi & 6.8005 & $::$ \\
74521 & 3465 & BI Cnc & A1 SiEuCr & 7.76 & vv \\
81009 & 3724 & KU Hya & A3 CrSrSi & 33.96 & v? \\
90044 & 4082 & SS Sex & B9 SiCrSr & 4.37894 & vv \\
116458 & 5049 & 67G. Mus & A0 SiEuCr & $(4.27:)$ & $? ?$ \\
119419 & 5158 & V827 Cen & A0 SiCrEu & 2.69562 & v: \\
125630 & GC 19369 & BS Cir & A2 SiCrSr & 2.205 & v: \\
137509 & GC 20810 & $\ldots$ & B9 SiCrFe & 4.4912 & vv \\
147010 & GC 21960 & V933 Sco & B9 SiCrSr & 3.920676 & vv \\
148199 & GC 22126 & $\ldots$ & B9 SiSr & 7.905 & v: \\
166469 & 6802 & V4045 Sgr & A0 SiCrSr & 2.88632 & v: \\
170397 & 6932 & V432 Sct & A0 SiCrEu & 2.19133 & vv \\
173650 & 7058 & V535 Her & A0 SiSrCr & 9.9754 & $:::$ \\
223640 & 9031 & ET Aqr & B9 SiSrCr & 3.735236 & vv \\
& & & & &
\end{tabular}

\begin{tabular}{rcclcc}
\hline 3980 & 183 & $\xi$ Phe A & A7 SrEuCr & 3.9516 & vv \\
4778 & 234 & G0 And & A1 CrSrEu & 2.562 & $:::$ \\
8441 & GC 1692 & HN And & A2 Sr & 69.5 & $:::$ \\
24712 & 1217 & DO Eri & A9 SrEuCr & 12.4610 & v: \\
49976 & 2534 & V592 Mon & A1 SrCrEu & 2.9760 & v: \\
51418 & GC 9158 & NY Aur & A0 HoDy & 5.438 & $:::$ \\
65339 & 3109 & AX Cam & A3 SrEuCr & 8.027 & $:::$ \\
72968 & 3398 & HY Hya & A2 SrCr & 11.305 & v? \\
83368 & 3831 & IM Vel & A8 SrCrEu & 2.851962 & $? ?$ \\
96616 & 4327 & V815 Cen & A3 Sr & 2.4394 & vv \\
98088 & 4369 & SV Crt & A8 SrCrEu & 5.90513 & vv \\
111133 & 4854 & EP Vir & A1 SrCrEu & 16.304 & $:::$ \\
118022 & 5105 & 78 Vir & A2 CrEuSr & 3.7220 & v: \\
125248 & 5355 & CS Vir & A1 EuCr & 9.29477 & vv \\
126515 & GC 19462 & FF Vir & A2 CrSr & 130.0 & $:::$ \\
128898 & 5463 & $\alpha$ Cir & A9 SrEu & & $? ?$ \\
137949 & GC 20814 & GZ Lib & F0 SrEuCr & $23.26:$ & $:::$ \\
148898 & 6153 & $\omega$ Oph & A6 SrCrEu & 0.74618 & v: \\
153882 & 6326 & V451 Her & A1 CrEu & 6.00890 & v: \\
164258 & 6709 & V2126 Oph & A3 SrCrEu & $0.359 / 0.719$ & v? \\
188041 & 7575 & V1291Aql & A6 SrCrEu & 5.438 & $:::$ \\
203006 & 8151 & $\theta^{1}$ Mic & A2 CrEuSr & 2.1221 & vv \\
220825 & 8911 & $\kappa$ Psc & A1 CrSrEu & 1.412 & $? ?$ \\
221760 & 8949 & $\iota$ Phe & A2 SrCrEu & $12.5:$ & v? \\
\hline \hline
\end{tabular}




\section{DISCUSSION}

The light variability of $\mathrm{CP}$ stars in the visible and in the ultraviolet has been ascertained in the past. On the basis of infrared photometry of $86 \mathrm{CP}$ stars, we have now established that these stars are also variable in the near infrared with the same period as visible light, spectrum, and magnetic field. This means that the properties which characterize CP stars (i.e. anomalous abundances, presence of magnetic fields, and variability) influence all parts of their spectra, although at a different rate, which is a surprising fact considering that the influence of the chemical peculiarities should gradually diminish in the infrared. Some regularities emerge from the bulk of data:

(i) Variations in the infrared have smaller amplitudes than in the ultraviolet and in the visible;

(ii) The infrared light curves do show nearly the same amplitude in all three $\mathrm{J}, \mathrm{H}, \mathrm{K}$ bands and the same shape and phase behavior with each other;

(iii) The extrema of the infrared light curves do not appear to correlate with the magnetic field polarity.

In a previous paper (CKL) we investigated the effects of high metallicity at the near infrared wavelengths and showed that a Kurucz model atmosphere with a metal content ten times the solar one could explain a three percent variation in the near infrared brightness, which is the typically observed value. Unfortunately Kurucz model atmospheres cannot exactly reproduce the flux distribution of magnetic CP stars but can describe their behavior at a qualitative level only, so, if larger variations are detected, we cannot exclude that other effects could be connected with the infrared variability. Among others, one such mechanism could originate from the presence of large-scale organized magnetic fields. The way in which a magnetic field may have influence on the conditions in a stellar atmosphere can be outlined in the three following:

i) modification (or even complete control) of the mechanisms which determine the formation of the chemical peculiarities;

ii) modification of the atmospheric structure of the star because of the contribution of the Lorentz force term in the hydrostatic equilibrium equation;

iii) modification of the radiation transfer in the lines through the Zeeman effect.

Item i) is important in determining the link between the geometry of the field and the nonhomogeneous distribution of the surface abundances.

Items ii) and iii) are more directly related to our problem of interpreting the suggested phase relation between the infrared light variation and the magnetic field extrema.

As far as it concerns item ii), the treatment of a model atmosphere in which a magnetic field is present is a very difficult problem: it has been discussed by some authors in some particular configurations (Trasco 1972, Staude 1972), but a more general approch has been carried out by Stepien (1978) who considered an 
essentially dipolar magnetic field slightly distorted by additional toroidal electric currents and calculated the stellar atmosphere structure taking into account the magnetic pressure term into the hydrostatic equilibrium equation. One of the most important results of Stepien's calculations is the star shape via the $\tau_{5000}$ parameter. According to the currents direction in the outermost layers, the star shape can be prolate or oblate with respect to the magnetic axis: the differences between the polar and the equatorial values of the radius being up to $3 \%$. The results obtained by Stepien lend support to a distorted figure of the star up to few percent and to small variations (2-3\%) of the effective temperature over the surface, which, in some cases, can contribute to the observed light variations.

Attempts have been made in the past to interprete the light variations of CP stars as a consequence of an oblate or prolate configuration: see, for example, Molnar (1974) for $a$ Cen and Böhm-Vitense \& Van Dyk (1987) for $\alpha^{2}$ CVn. This explanation alone is not sufficient as far as it concerns the many stars which show different behavior in the $u, v, b, y$ light curves. However, since the magnetic pressure importance increases in the outer layers, it cannot be excluded that the non-spherical shape of the star as seen at the infrared wavelengths is the origin of the observed variability.

At the moment the observational data are not sufficient to investigate further the problem, especially because the period of too many stars is not known with the requested accuracy to derive accurate phase relations for data taken several years apart, but we think that the study of the relation between the infrared and magnetic variations may supply valuable information to understand the puzzling problem of magnetic CP stars.

\section{REFERENCES}

Böhm-Vitense E., Van Dyk S.D., 1987, AJ 93, 1527

Bouchet P., 1989, Infrared Photometry, E.S.O. Operating Manual N.11

Catalano F.A., 1975, Mem. Soc. Roy. Sci. Liège 7, 117

Catalano F.A., Kroll R., Leone F., 1991a, A\&A 248, 179 (CKL)

Catalano F.A., Leone F., 1992, A\&AS (in press)

Catalano F.A., Renson P., 1984, A\&AS 55, 371

Catalano F.A., Renson P., 1988, A\&AS 72, 1

Catalano F.A., Renson P., Leone F., 1991b, A\&AS 87, 59

Catalano F.A., Renson P., Leone F., 1992, A\&AS (to be submitted)

Kroll R., Schneider H., Catalano F.A., Voigt H.H., 1987, A\&AS 67, 195

Leone F., Catalano F.A., 1991, A\&A 242, 199

Manfroid J., Sterken C., Bruch A., et al., 1991, First Catalogue of stars measured in the Long-Term Photometry of Variable project 1982-1986,

E.S.O. Scientific Report N.8

Molnar M.R., 1974, ApJ 187, 531

Preston G.W., 1974, ARA\&A 12, 257

Renson P., Gerbaldi M., Catalano F.A., 1991, A\&AS 89, 429

Stepien K., 1978, A\&A 70, 509

Trasco J.D., 1972, ApJ 171, 569 


\section{DISCUSSION (Catalano, Leone and Kroll)}

SMITH: Did you consider fitting line profiles rather than equivalent widths? And is there more information in the line profiles than in the equivalent widths? KROLL: There is certainly more information in the line profile than in the equivalent width. I fitted the line profiles with Peterson's BALMER models with $10 \times$ solar metals. However, it is usually not possible to fit $\mathrm{T}_{\text {eff }}$ and $\log g$ simultaneously. You need to have more information to restrict one of those parameters. ADELMAN: Is $B D+24^{\circ} 3675$ a known variable?

KROLL: The period is about 7.52 days, as measured by Schneider (1986, IBVS 2870). 